\title{
Vibration Suppression with Resettable Device
}

\author{
Faryar Jabbari ${ }^{1}$ and James E. Bobrow ${ }^{2}$
}

\begin{abstract}
Novel low-power or semiactive devices are developed for vibration suppression applications. By manipulating the structural stiffness, the resisting forces generated by the devices are quite large and independent of velocity. Experimental results are presented to demonstrate the feasibility of the devices and to justify the main assumptions used. The critical design issue of device placement is addressed analytically and verified. While much of the development here applies to the case of variable stiffness devices, we focus on resettable devices in which the device behaves like a linear spring. However, at appropriate times, the effective unstretched length of the device is changed - or reset - to extract energy from the vibrating structure.
\end{abstract}

DOI: 10.1061/(ASCE)0733-9399(2002)128:9(916)

CE Database keywords: Vibration control; Stiffness.

\section{Introduction}

Low-power devices that can act as actuators in a variety of vibration suppression applications have been receiving increasing attention. With a slight abuse of notation, these are often called semiactive devices due to their very low power consumption (often provided by compact batteries), even though they contain sensors and a control logic that governs the overall behavior of the device. They typically cannot add energy to the system (hence, "semiactive"), are subject to a set of decentralized control logic, and have superior reliability, particularly when compared to traditional actuators.

The benefits of relying on such low-power (or semiactive) devices have been shown in a variety of applications. While the literature is quite extensive and a comprehensive survey is not feasible, applications to structures can be found in Kobori and Kamagata (1991), while Onodo and Minesugi (1996a,b) discuss applications to space structures. The work in Patten and Sack (1994) and Patten et al. (1996) shows the benefits in bridge applications, while Bobrow et al. (2000) discuss the benefits in shock absorbers. While earlier devices were primarily hydraulic (see, for example, Taylor and Constantinou 1995), recent progress in electrorheological and magnetorheological materials has lead to a variety of new semiactive devices employing such materials. A large number of references (see Carlson et al. 1995; McClamroch and Gavin 1995; Gavin 1997; Gordaninejad and Breese 1999 as a representative sample) discuss the benefits and shortcomings associated with each technique, and several different approaches are used for control. Some are primarily variable stiffness techniques, which may or may not match the semiactive nature of the

\footnotetext{
${ }^{1}$ Dept. of Mechanical and Aerospace Engineering, Henry Samueli School of Engineering, Univ. of California, Irvine, CA 92697 (corresponding author). E-mail: fjabbari@uci.edu

${ }^{2}$ Dept. of Mechanical and Aerospace Engineering, Henry Samueli School of Engineering, Univ. of California, Irvine, CA 92697.

Note. Associate Editor: James L. Beck. Discussion open until February 1, 2003. Separate discussions must be submitted for individual papers. To extend the closing date by one month, a written request must be filed with the ASCE Managing Editor. The manuscript for this paper was submitted for review and possible publication on March 30, 2001; approved on January 3, 2002. This paper is part of the Journal of Engineering Mechanics, Vol. 128, No. 9, September 1, 2002. ()ASCE, ISSN 0733-9399/2002/9-916-924/\$8.00+\$.50 per page.
}

devices (e.g., Leitmann and Reithmeiren 1993; Onodo and Minesugi 1996a; Nagarajaiah 1997 etc.). Others devise control logic that explicitly incorporate the semiactive concept (e.g., Nemin et al. 1994; Patten et al. 1996 among many). Often, the logic serves as an approximation of a more traditional control technique (see Onodo and Minesugi 1996a or the clipped optimal control in Dyke et al. 1996 or Spencer et al. 1997).

Here, we focus on a new class of semiactive devices. Theses devices, which were introduced in Bobrow et al. (1995), offer several advantages. Unlike most, though not all, of the work reported in literature, the proposed devices manipulate the stiffness properties and are capable of producing large resisting forces. As shown in Bobrow et al. (2000), the lack of dependence on velocity also contributes to beneficial response in "shock"-type disturbances, since the force transmitted through the device is considerably smaller than those in traditional devices. The basic design is feasible for both pneumatic and hydraulic implementation, as shown below, and employs relatively simple mechanisms and control logic. It offers a great deal of reliability due to its reliance on standard hydraulic or pneumatic concepts, particularly when compared to devices employing novel material. Naturally, it possesses the low-power, semiactive, and decentralized properties that many of these devices share. Some of the benefits and advantages of the resetting devices, as compared to other semiactive approaches, are described in Yang and Agrawak (2001).

In the next section, we briefly review the basic concept, hardware design, and preliminary results. Next, we present results that offer evidence regarding the feasibility of the assumptions used in developing a model for these devices and show experimental results obtained from prototypes. A subsequent section deals with the stability results for the multidegree of freedom systems, as well as results for placement of these devices. Finally, simulation and experimental results are used to establish the validity and the benefits of the proposed techniques.

\section{Background and Preliminaries}

We focus on a new class of devices that alter the stiffness characteristics of the system, compared to most of the devices (e.g., electrorheological) that affect the damping properties. The essential properties are captured in the schematic of Fig. 1, in which the device is connected to a one-degree-of-freedom mass spring 


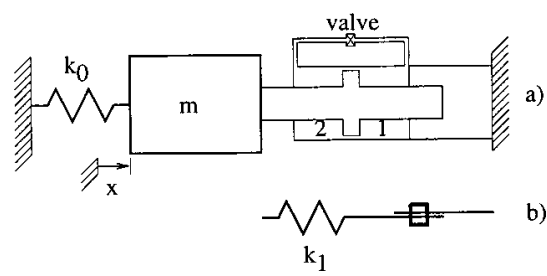

Fig. 1. Schematic of semiactive element

system. The device is essentially a piston, a double acting cylinder and a valve. The valve connects the two sides of the cylinder. When it is closed, the fluid in the cylinder is compressed due to the movement of the piston. Pneumatic and hydraulic versions of the semiactive device are shown in Fig. 2. The effect of compression can be approximated by a linear spring (see the next section for details). The device can thus provide large resisting forces. Once the valve is open, the energy stored in the fluid is turned into heat and is dissipated (by slushing and internal damping). Schematically, the closed valve corresponds to adding stiffness to the system (e.g., $k_{1}$ in Fig. 1), while opening of the valve corresponds to removing stiffness and extracting the potential energy stored in $k_{1}$. It is assumed that the valve orifice is large enough so that no force is generated from the device as the piston moves. This means that the device does not add velocity-dependent damping to the system.

Two general approaches can be considered.

1. Resetting technique: In this approach, the valve can be opened at any time to extract energy from the system, but it is then closed as soon as the pressure difference across the piston is eliminated. Schematically, this is equivalent to "resetting" the spring to the higher stiffness value as soon as the strain energy in the spring is converted into heat. Since the "reset time" is short, instead of modeling a variable stiffness, the resulting device exhibits the high-stiffness value at all times, and at the switching times "resets" the unstretched length of the equivalent spring element.

2. Semiactive variable stiffness technique: In this approach, the device operates at two distinct stiffness levels. The valve can be opened (for low stiffness) at any time to extract energy, but it is changed to high stiffness (by closing the valve) only when the system passes through the zero position. This last requirement is needed to apply the traditional variable stiffness approach (Nemin et al. 1994; Utkin 1997) while maintaining the semiactive nature of the hardware. That is, increasing the stiffness of a spring requires the input of energy unless it is done at its unstretched position.

In a simple single-degree-of-freedom system, the standard variable stiffness approach employs the following control logic: When $x \dot{x} \geqslant 0$, the valve is closed, resulting in the higher stiffness (in Fig. 1, stiffness is $k_{0}+k_{1}$ ) and when $x \dot{x}<0$ the valve is opened, resulting in the lower stiffness (stiffness is $k_{0}$ ). Alterna-


Fig. 2. Prototype devices: pneumatic (left) and hydraulic (right)
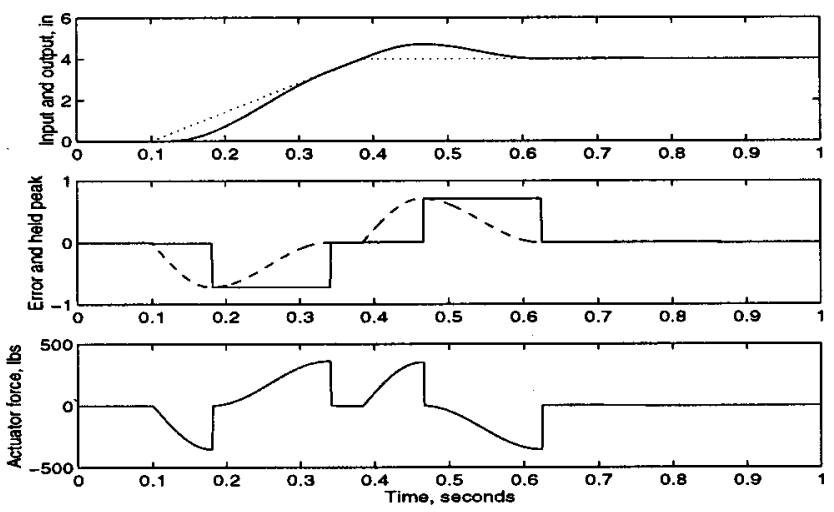

Fig. 3. Simulink response of mass spring system with peak detection and resetting control

tively, the resetting approach is based on the following logic: the value is closed at all times, except when $\dot{x}=0$. In that case, the valve is open to extract energy and closed as soon as possible. Considering the equivalent "spring" analogy, this implies that when $\dot{x}=0$, the unstretched length of the spring $\left(x_{s}\right)$ is reset, i.e.,

$$
\text { set } x_{s}=x \text { whenever } \dot{x}(t)=0 \text {, and }|x(t)|>\epsilon
$$

where the condition on magnitude of $x$ is for avoiding chattering when $x(t)$ decays to very small values. In this setting, the equation of motion for the single-degree-of-freedom system is

$$
m \ddot{x}+\left(k_{0}+k_{1}\right) x=k_{1} x_{s}
$$

Note that the structural stiffness is always at its maximum value, and that the reset times are assumed to be instantaneous. As discussed in Bobrow et al. (1995, 2000), this control logic is motivated by the observation that once the sign of $\dot{x}$ changes, the device is transferring the stored stain energy back to the system, and resetting it according to the logic above extracts the energy and prevents this transfer.

The advantages of the resetting approach over variable stiffness is due to several issues. By providing high stiffness, reliability and safety are not compromised and the resisting force is always at its maximum value. Furthermore, the number of times energy is extracted is higher in the resetting technique, leading to faster and more effective vibration suppression. In the simple single degree of freedom in Fig. 1, for example, it was shown in Bobrow et al. (1995) that the rate of decay is $\left(k_{0}-k_{1} / k_{0}\right.$ $\left.+k_{1}\right)^{n_{1}}$, where $n_{1}$ is the number of peaks or reset times. The rate of decay for the variable stiffness is $\left(k_{1} / k_{0}-k_{1}\right)^{n_{2}}$ where $n_{2}$ is number of peaks (which is half of $n_{1}$ ). Naturally, resetting results in much faster decay. If $k_{0}=k_{1}$, for example, the system comes to a stop in less than one period. The final advantage of the resetting approach is that it maintains the natural frequency and mode shapes of the system. This property, which is also called the homogeneity in Inaudi et al. (1994), will be used in the section, "Device Model and Experimental Verification," to establish stability and device location results. While most of the techniques discussed here also apply to variable stiffness devices, for brevity we focus on resetting devices.

\section{One Degree of Freedom Example: Shock Absorber}

The resetting-type control law also has outstanding shock isolation characteristics. As an example, consider the case of a car 

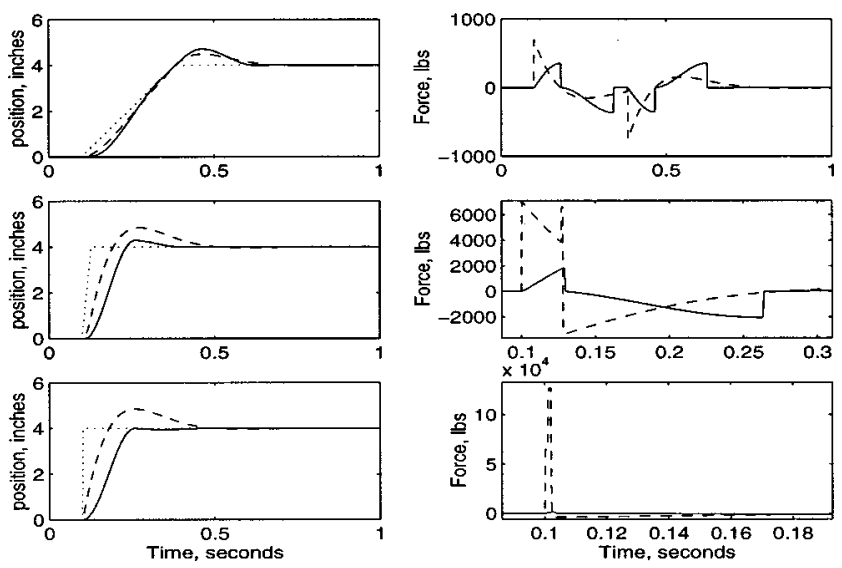

Fig. 4. Simulation for three velocities

going over a ramp-shaped bump. The car is approximated by a mass-spring system with a base displacement $z(t)$ as input. The ramp shaped input function is 4 -in. high and 5 -in. wide. The new equation of motion has the same form as before, but the position $x$ must be replaced by the relative displacement $x-z$ for the two stiffness terms. The results for a 1,000 lb, 1/4 car model traveling over the bump at $1 \mathrm{mph}$ are shown in Fig. 3. The spring constants were $k_{0}=k_{1}=500 \mathrm{lb} / \mathrm{in}$. The top plot shows the input (dotted) and output (solid) positions as functions of time. The middle plot shows the spring deformation $x-z$, referred to as the "error" in the plot (dotted), and the value of $x_{s}$ used by the resetting control law (solid, referred to as held peak). Note that the changes in the unstretched spring length $x_{s}$ occur at times when $\dot{x}-\dot{z}=0$. The bottom plot shows the force in the device. Note that at the four instants when the force reaches its peak value, all the energy stored in the device is discarded and the process repeats.

More results of the simulation are shown in Fig. 4 for three cases when the mass is traveling at velocities of 1,10 , and 100 mph. The solid lines in the left-hand plots are the displacement of the mass with the proposed device, the dotted lines are the bump, and the dashed lines are the response of the mass spring system with a conventional viscous damper with $\zeta=0.707$. The comparison is thus between the shock isolation features of a conventional damper used in conjunction with the stiffness of $k_{0}$ to those of the resettable device with stiffness $k_{1}$. The interesting feature of the comparison is that the conventional damper transmits a large shock load to the mass due to the sudden base velocity when the bump is encountered. At high speeds, the shock load is extremely large and causes the mass to overshoot the final displacement. The right-hand plot shows the resisting force in the viscous damper (dashed) and in the proposed device (solid). The time scales have been adjusted in these plots. For the case of $10 \mathrm{mph}$, the peak force produced by the viscous damper on the mass is 3.4 times that of the proposed device. At $100 \mathrm{mph}$, the force is 63 times more in the viscous damper. These results indicate a clear advantage of the proposed device over conventional dampers when shock loads are present.

\section{Device Model and Experimental Verification}

In this section, we discuss prototypes of devices that meet the two critical assumptions needed for the techniques used here: behavior similar to a linear spring, and the ability to be "reset" (i.e., to rapidly extract the energy from the device and then to return to a high stiffness). There are several hardware designs that could be used to obtain these basic features. The choice of the device depends on the force and stiffness requirements. For relatively low stiffness, a simple and reliable actuator can be obtained by using a gas such as air as the working fluid in the device. As shown below, air can achieve a wide range of effective stiffness $k_{1}$ values. Another possibility is to use a hydraulic fluid in the device, and choose the piston area that achieves a fluid compressibility that matches $k_{1}$. A third method is to use a hydraulic fluid, but choose the piston area large enough to ensure that the fluid does not compress at all. Then an external spring of stiffness $k_{1}$ can be placed in series with the device to achieve the desired effect.

Because of its simplicity, we will discuss the design of a pneumatic device. We will first determine the relationship between the cylinder dimensions and the stiffness $k_{1}$. Let the pressure on the left-hand side of the device in Fig. 1 be $p_{2}$, and the pressure on the right-hand side be $p_{1}$. Then the force on the mass is $F$ $=\left(p_{2}-p_{1}\right) A$, where $A$ is the piston area. Assuming an ideal gas
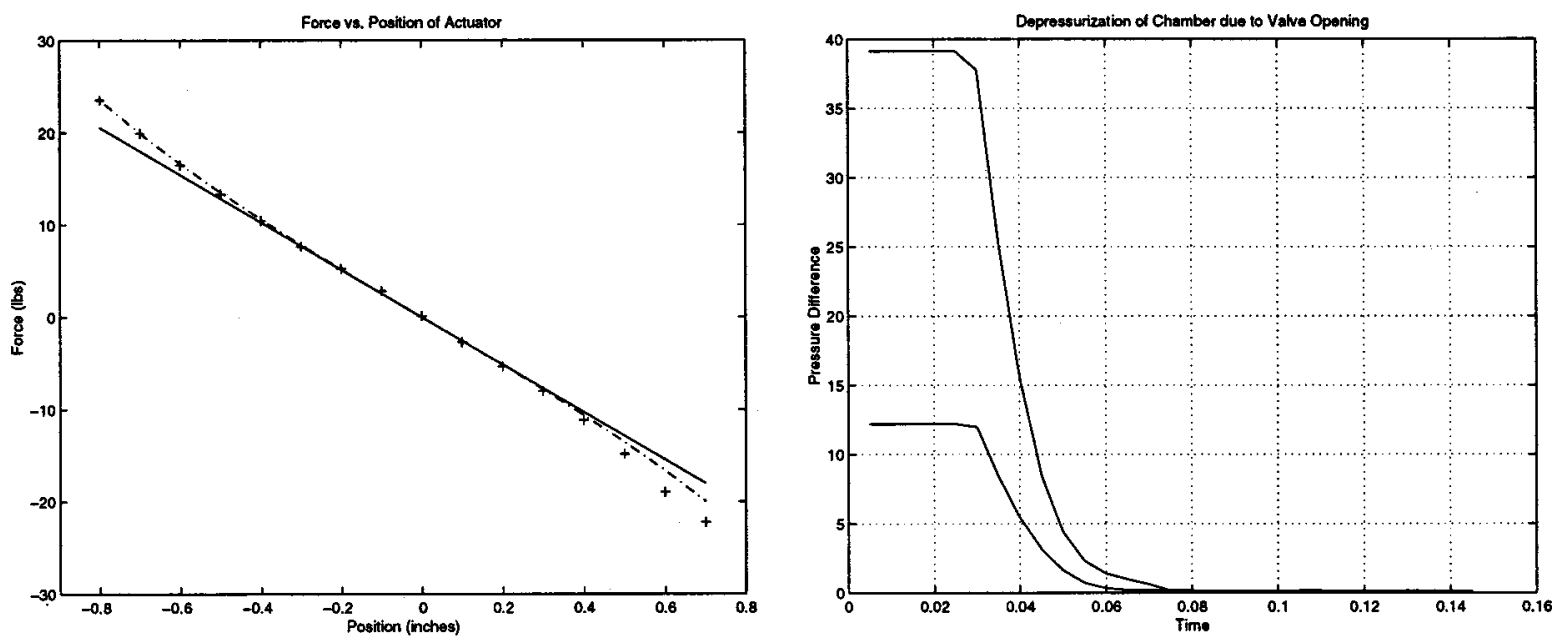

Fig. 5. Left plot shows approximate linearity between force versus displacement. Right plot shows the experimentally measured time to "reset" device from two initial pressures. 
with no heat transfer through the cylinder walls, the pressures on both sides of the cylinder are governed by isentropic compression $p_{i} V_{i}^{\gamma}=c ; i=1,2$ where $\gamma$ is the ratio of specific heats $(\gamma=1.4$ for air), $V_{i}$ are the volumes on the two sides of the cylinder, and $c$ is a constant. Assuming we start motion from the midstroke position with the initial pressures on both sides of the cylinder equal to $p_{0}$ and initial volumes $V_{0}$, with $p_{0} V_{0}^{\gamma}=c$, we have

$$
F(x)=\left(p_{2}-p_{1}\right) A=\left[\left(V_{0}+A x\right)^{-\gamma}-\left(V_{0}-A x\right)^{-\gamma}\right] A c
$$

where we have let the volumes on both sides of the cylinder change with the cylinder position $x$. A local approximation for the effective spring constant of the gas and cylinder is obtained if one linearizes Eq. (2) for small motions of $x$. The result is

$$
F(x)=-\frac{2 A^{2} \gamma p_{0}}{V_{0}} x
$$

Hence, the effective spring constant $k_{1}$ is $k_{1}=2 A^{2} \gamma p_{0} / V_{0}$.

This relationship was tested experimentally on a resettable device with a bore of $1.5 \mathrm{in}$. and a stroke $=2.8 \mathrm{in}$. (see the prototype depicted on the left-hand side of Fig. 2). During the experiment, the piston was moved approximately 1 in. inwards and 1 in. outwards starting from the midstroke position. The force was measured at 0.1 intervals with a force transducer. The resulting data are shown in Fig. 5. The points labeled with + are the experimental measurements. The linear plot is from Eq. (3) and the dashed plot is from Eq (2). Observe that, over the range of motion used, the linear approximation of the device's behavior is justified.

The second test performed concerned the "resetting" property of the device. In this test, the solenoid valve that connects the two sides of the cylinder was opened and the pressures in the two sides of the cylinder were recorded. In the ideal case, the pressure difference would immediately reach zero. This would mean that the internal energy of the gas would be irreversibly lost to heat since the entropy is increased during the expansion. If the valve were then closed, the current location of the piston would be the new zero position of the gas "spring." However, since the orifice in the valve is relatively small, it takes some time for the gas to flow between the two sides of the cylinder and reach equilibrium. The response time shown in Fig. 5 is thus due to the time it takes to fully open the valve and to the gas dynamics. The time delay or lag shown in Fig. 5 (approximately 20-30 ms) is small enough for systems with frequencies up to about $20 \mathrm{~Hz}$ such as most structures and automotive suspensions. Systems with higher frequencies would require faster valves and larger orifices to achieve faster reset rates. As discussed in Thai (1997), the exponentiallike decay seen in Fig. 5 can also be used in the control law development.

For higher capacity, hydraulic devices can be developed. In Bobrow et al. (1995), we show that the stiffness created by a hydraulic system is $k_{1}=4 A \beta / s$, where $\beta$ is the fluid bulk modulus and $s$ is the cylinder stroke length. The device shown on the right-hand side of Fig. 2 is a prototype that has a stiffness of $2,000 \mathrm{lb} / \mathrm{in}$. It is straightforward to create devices with output forces in the megaton range, if required.

\section{Multidegree of Freedom Systems}

\section{Modeling}

The basic idea discussed in the section, "Background and Preliminaries," above can be generalized to multidegree of freedom systems. Consider an $n$ degree-of-freedom system with $x$ as the vector of generalized coordinates. Assume that there are $l$ devices. The energy stored in the devices is the following:

$$
U_{a}=\sum_{i=1}^{l} U_{i}=\frac{1}{2} \sum_{i=1}^{l}\left(x-x_{s, i}\right)^{T} K_{i}\left(x-x_{s, i}\right)
$$

In Eq. (4), $x_{s, i}=$ piece-wise continuous vector denoting the most recent zero force position of the $i$ th device (i.e., the value of $x$ at the last resetting of the device) and $K_{i}=$ stiffness matrix associated with the $i$ th device. For the devices discussed earlier, each $K_{i}$ is a rank-one matrix and can be written as

$$
K_{i}=v_{i} v_{i}^{T} \alpha_{i}
$$

where $\alpha_{i}=$ stiffness of the device and $v_{i}=$ vector that depends on the location of the device. While $v_{i}$ can always be obtained through a simple singular value decomposition, in practice, it often can be written by inspection. This is due to the fact that each device often connects a very limited number of degrees of freedom and, as a result, $K_{i}$ is often a sparse matrix with a few entries. For example, consider the relative displacement of the ends of the $i$ th device, $z_{i}$ with the corresponding zero force position of $z_{s, i}$. Then, it is straightforward to see that

$$
z_{i}-z_{s, i}=v_{i}^{T}\left(x-x_{s, i}\right)
$$

i.e., $v_{i}=$ vector of transformation from the general coordinates to those based on the device.

Adding Eq. (4) to the total potential energy, we obtain the following for the total mechanical energy of the system:

$$
\begin{aligned}
E & =\frac{1}{2} \dot{x}^{T} M \dot{x}+\frac{1}{2} x^{T} K x+\frac{1}{2} \sum_{i=1}^{l}\left(x-x_{s, i}\right)^{T} K_{i}\left(x-x_{s, i}\right) \\
& =T+V+\sum_{i=1}^{l} U_{i}(t)
\end{aligned}
$$

The first term=kinetic energy; the second term=stain energy due to the nominal stiffness of the system; while the last term=energy stored in the resettable devices. As shown below, the first two terms will be used as a Lyapunov candidate to establish the stability of the system. Application of Lagrange's equations leads to the following for the equations of motion for a multidegree of freedom system

$$
M \ddot{x}+K x+\sum_{i=1}^{l} K_{i}\left(x-x_{s, i}\right)=0
$$

where $M$ and $K=$ nominal mass and stiffness matrices of the structure (i.e., without the resettable devices). Naturally, damping would add another term (e.g., $C \dot{x}$ ) to Eq. (7).

Next consider the energy stored in each device $U_{i}$ [see Eq. (4)]. Once the device is reset, i.e., $x_{s, i}$ is set to the current value of $x$, all of the energy is extracted and from that point on, any displacement results in the device collecting energy (from the system). As long as the device is storing energy, $\dot{U}_{i}>0$, while $\dot{U}_{i}$ $<0$ denotes the case where energy is leaving the device (back to the system). This observation leads to the following control logic, which seeks to ensure that the device always takes energy from the system by resetting it as soon as $\dot{U}$ is reduced to zero

$$
\text { set } x_{s, i}=x \text { whenever } \dot{U}_{i}=0, \text { for } i=1,2, \ldots, l
$$

where $U_{i}$ is from Eq. (4) and thus

$$
\dot{U}_{i}=\dot{x}^{T} K_{i}\left(x-x_{s, i}\right)
$$



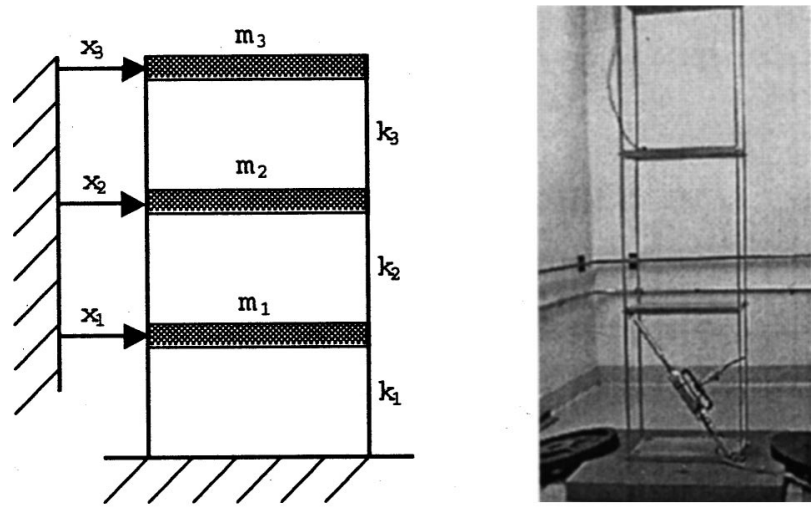

Fig. 6. Three bay scale structure and its schematic

Using the coordinates that represent relative displacements between the ends of the device (i.e., $z_{i}$ discussed above), the control logic becomes

$$
\text { set } z_{s, i}=z_{i} \text { whenever } \dot{z}_{i} \alpha_{i}\left(z_{i}-z_{s, i}\right)=0
$$

Note that due to resetting, beyond the trivial case of $z_{i}=\dot{z}_{i}$ $=0$, the only times we can encounter $\dot{U}_{i}=0$ is when $\dot{z}_{i}=0$, i.e., when the relative motion of the ends of the device is changing direction. The basic idea is essentially the same as the single degree of freedom system, which is also derived with similar motivations. The control logic is decentralized since it requires only local information (i.e., resetting of $i$ th device depends on the derivative of the relative displacement of $i$ th device only). Finally, note that Eq. (9) does not depend on stiffness, mass, or damping matrices and, as such, is robust with respect to modeling errors in these matrices.

Before discussing stability or placement issues, we can briefly discuss the variable stiffness approach. In this approach, the device is either set to low (i.e., $\left.\alpha_{i}=0\right)$ or high (i.e., $\alpha_{i} \neq 0$ ). There is no resetting and thus $z_{s, i}=0$ (or $x_{s, i}=0$ ). Using the same physical motivation as above, as long as the device can store energy (thus extracting energy from the system), the device is set to high stiffness. A positive rate of energy storage, along the lines used above, leads to

$$
K_{i}=\alpha_{i} v_{i} v_{i}^{T}(\neq 0) \quad \text { if } \dot{z}_{i} z_{i} \geqslant 0
$$

otherwise (i.e., $\left.\dot{z}_{i} z_{i}<0\right)$ the valve is open $\left(K_{i}=0\right)$. The switch to low stiffness occurs when $\dot{z}_{i}=0$ (maximum deflection), and the

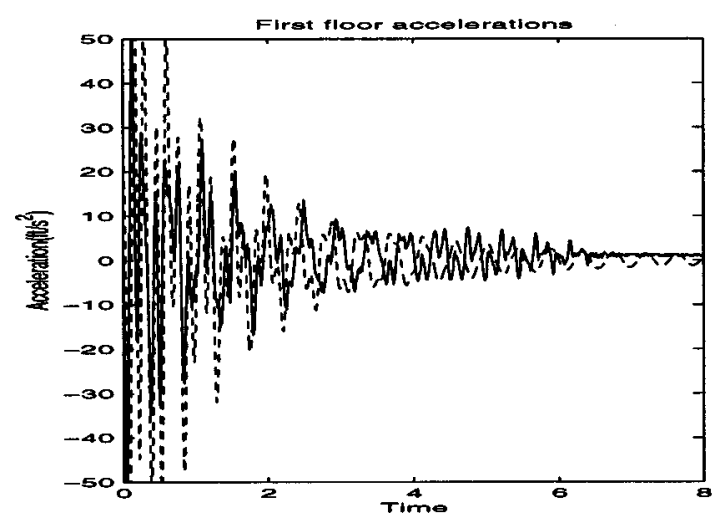

switch to high stiffness occurs when $z_{i}=0$. Note that since stiffness is increased at $z_{i}=0$, no energy is required (beyond perhaps closing a small valve). The equations of motion become more complicated, however

$$
M \ddot{x}+\left(K+K^{*}\right) x=0
$$

where $K^{*}$ has the form

$$
K^{*}=\sum_{i=1}^{l} \beta_{i} K_{i}, \quad \beta_{i}=0 \quad \text { if } \dot{z}_{i} z_{i}<0, \quad i=1,2, \ldots, l
$$

\section{Stability}

For brevity, we will primarily discuss the resetting technique, though extensions and modifications needed for the variable stiffness technique will be mentioned briefly. We start with the total mechanical energy of the system, without the semiactive devices, as the Lyapunov candidate [see Eq. (6) above]. Following standard arguments (see, e.g., Slotine and Li 1991 or Khalil 1996), we need to show $\dot{V} \leqslant 0$ to establish stability in the sense of Lyapunov. Taking the derivative of $V$ along the solutions to Eq. (7), we obtain $\dot{V}=-\Sigma \dot{U}_{i}$. The resetting logic guarantees that $\dot{U}_{i} \geqslant 0, \forall_{i}$, since each device is always absorbing energy from the system (and is reset as soon as $\dot{U}_{i}=0$ ). We thus have $\dot{V} \leqslant 0$ which establishes Lyapunov stability and boundedness of the state vector. Note that this reasoning is easily modified for the variable stiffness technique.

In general, the results above do not establish asymptotic stability (i.e., we cannot guarantee that the entire state vector goes to zero). Indeed, it is easy to construct simple (undamped) multidegree-of-freedom systems with certain initial conditions, in which the state vector does not go to zero (more on this later). By considering the system an autonomous nonlinear (state-dependent stiffness) system, standard application of LaSalle's theorem (Slotine and Li 1991) yields $\lim _{t \rightarrow \infty} \dot{x}^{T} K_{i}\left(x-x_{s, i}\right)=0$. Using the local $z$ coordinates, this implies that $\dot{z} \rightarrow 0$, which means that the relative motion of the ends of each device goes to zero. Next, denoting

$$
S_{i}(t)=\dot{x}^{T}(t) K_{i} \dot{x}(t)
$$

this implies that

$$
\lim _{t \rightarrow \infty} S_{i}(t)=0, \quad \forall i
$$

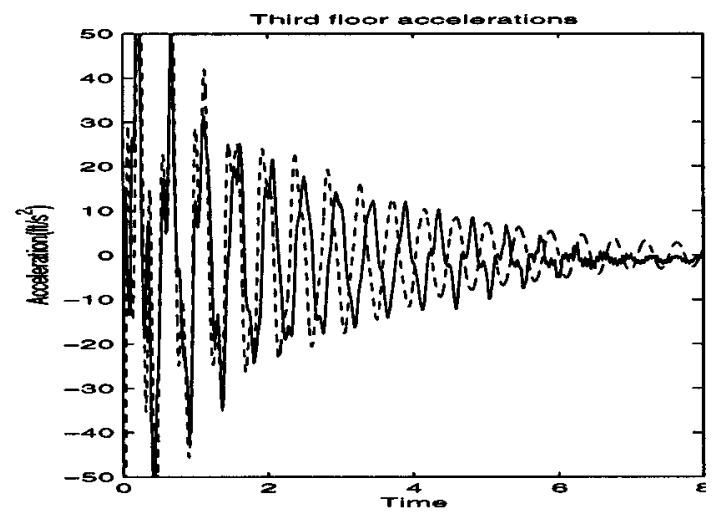

Fig. 7. Absolute acceleration of floor one (left) and floor three (right) due to initial displacement on floor three: Solid line is experimental data and dashed line is from simulation 

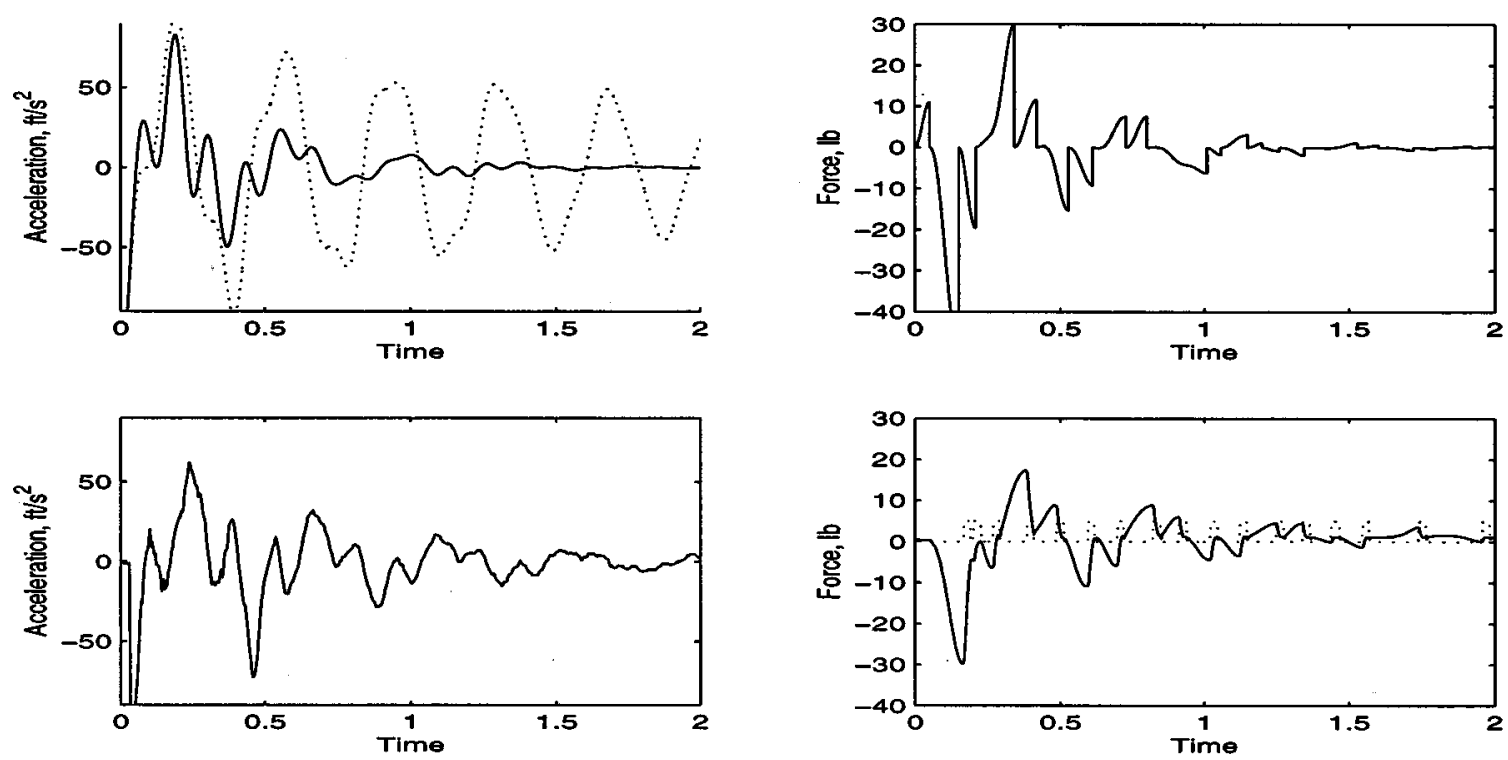

Fig. 8. Simulated (upper) and experimental (lower) results for acceleration of floor three and resisting force of device

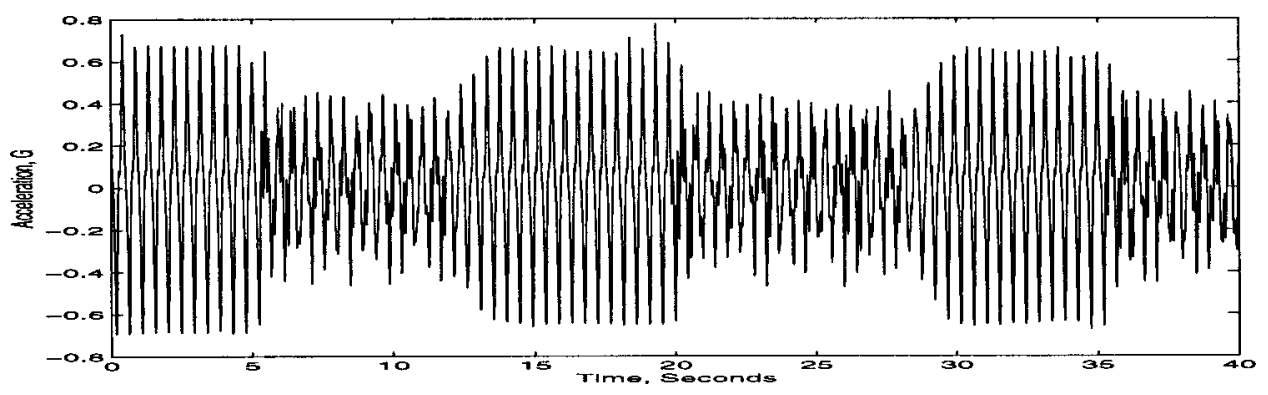

Fig. 9. Acceleration of top floor with periodic input near first resonant frequency, with and without control

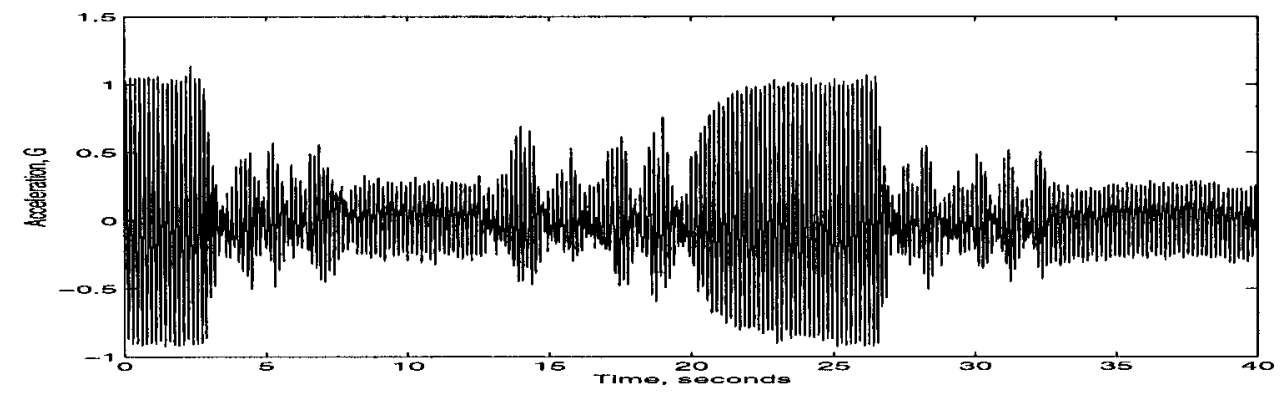

Fig. 10. Acceleration of top floor with periodic input near second resonant frequency, with and without control

Putting all $l$ devices together, we have the state vector $x$ converging to the intersection of all $S_{i}=0$. Recall that if there were damping in the system, through a $C \dot{x}$ term in Eq. (7), similar arguments would lead to $\lim _{t \rightarrow \infty} \dot{x}^{T} C \dot{x}=0$. As a result, we have

$$
\lim _{t \rightarrow \infty} \dot{x}(t) \in S(t)
$$

where

$$
S(t)=\left\{S_{1}(t)=0\right\} \cap\left\{S_{2}(t)=0\right\} \cdots \cap\left\{\dot{x}^{T} C \dot{x}=0\right\}
$$

As before, note that with minor alteration, similar conclusions can be obtained for the variable stiffness approach as well. In either case, the control logic leads to $\dot{z}_{i} \rightarrow 0$, but not necessarily to either $x$ or $\dot{x}$ becoming zero. Indeed, it is easy to construct simple systems that show this. For example, consider an undamped two- mass system with a semiactive device connecting the two masses. It is relatively straightforward to verify that the semiactive device eliminates all relative motion between the two masses, but the two masses may move together based on initial conditions and how they are anchored to the support. For the same system, however, a semiactive device connecting one of the masses to the support would eventually eliminate all motion. This observation points to the important role that the placement of these devices play.

\section{Device Placement}

We start with the standard diagonalization through the eigenvectors of the $M$ and $K+\Sigma_{i} K_{i}$ system, i.e., the stiffness of the system 


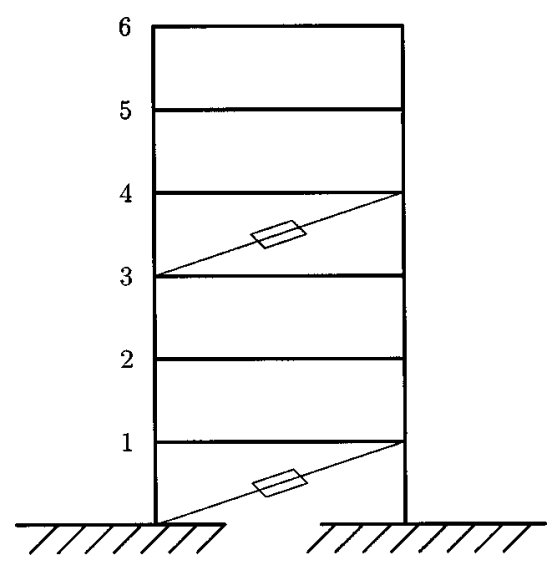

Fig. 11. Schematic of six-story building

when all of the devices are set to the high-stiffness state. Let matrix $Q$ be the eigenvector matrix along with standard change of coordinates

$$
x(t)=Q y(t)
$$

which leads to the following equations of motion in terms of the modal coordinates:

$$
m_{j} \ddot{y}_{j}(t)+\lambda_{j} y_{j}(t)=q_{j}^{T} \sum_{i} K_{i} x_{s, i}=\sum_{i} q_{j}^{T} v_{i} \alpha_{i}\left(v_{i}^{T} x_{s, i}\right)
$$

where $m_{j}$ and $\lambda_{j}=$ generalized mass and stiffness for the $j$ th mode; and $q_{j}=$ corresponding eigenvector. Recalling Eq. (5) regarding the stiffness associated with each device, it is easy to see that $q_{j}^{T} v_{i} \alpha_{i}$ plays a role similar to the $k_{i}$ in Eq. (1) for the single degree of freedom discussed earlier. As a result, if $v_{i}^{T} q_{j}=0$ then the device installed on the $i$ th floor does not have any impact on the $j$ th mode. Typically, the larger the $v_{i}^{T} q_{j}$, the faster the vibration in the $j$ th mode is eliminated. This can be used to help identify desirable locations for the devices (e.g., placed such that highly excited modes are the ones most affected). This issue will be considered further below.

The placement issue discussed above relied on the general concept of mode shapes, which is possible due to the homogeneity (Inaudi et al. 1994) property of these classes of nonlinear systems (e.g., the stiffness is the same high value at all times). Unlike previous results, it is not clear how these pole placement results apply to the variable stiffness approach. Recall that in that case, the stiffness matrix is only piece-wise constant and the concept of natural frequency and mode shapes do not apply. Attempting diagonalization with the nominal mode shapes can lead to weaker results that show that if a mode shape is perpendicular to all $v_{i}$ 's,
Table 1. $v_{i}^{T} q_{j}$ for Different Mode Shapes and Device Locations

\begin{tabular}{lrrrrrr}
\hline $\begin{array}{l}\text { Floor } \\
i\end{array}$ & $\begin{array}{c}\text { First } \\
\text { mode }\end{array}$ & $\begin{array}{c}\text { Second } \\
\text { mode }\end{array}$ & $\begin{array}{r}\text { Third } \\
\text { mode }\end{array}$ & $\begin{array}{r}\text { Fourth } \\
\text { mode }\end{array}$ & $\begin{array}{r}\text { Fifth } \\
\text { mode }\end{array}$ & $\begin{array}{r}\text { Sixth } \\
\text { mode }\end{array}$ \\
\hline 1 & 0.1327 & -0.3678 & 0.5187 & 0.5507 & -0.4565 & -0.2578 \\
2 & 0.1250 & -0.1828 & -0.1508 & -0.6834 & 0.9752 & 0.7143 \\
3 & 0.1101 & 0.0941 & -0.6256 & -0.3859 & -0.6514 & -1.0072 \\
4 & 0.0887 & 0.3238 & -0.2929 & 0.7764 & -0.2351 & 1.0693 \\
5 & 0.0621 & 0.3905 & 0.4179 & 0.1987 & 0.9185 & -0.8865 \\
6 & 0.0320 & 0.2609 & 0.5893 & -0.8243 & -0.8084 & 0.5006 \\
\hline
\end{tabular}

then that mode is not affected by the device and undergoes free vibration. In general, however, the modal coordinates cannot be separated.

\section{Results}

Consider the scale model of a three-story (or three-bay) structure in Fig. 6. On the right is the laboratory model from which the data presented here are obtained, and on the left is the schematic of the equivalent three-degree-of-freedom model used to represent the structure

$$
M \ddot{x}+C \dot{x}+K x=B w
$$

where $x=$ vector containing displacements of floors, $w=$ disturbance (e.g., a rotating unbalance on the top floor), and $M, C$, and $K=$ mass, damping, and stiffness matrices, respectively. Structural identification (Srisamang 1997) leads to the following values for these matrices:

$$
\begin{aligned}
M & =\left[\begin{array}{lll}
0.233 & 0 & 0 \\
0 & 0.233 & 0 \\
0 & 0 & 0.233
\end{array}\right] \\
C & =\left[\begin{array}{rrr}
0.523 & -0.153 & -0.035 \\
-0.153 & 0.502 & -0.200 \\
-0.035 & -0.200 & 0.353
\end{array}\right] \\
K & =\left[\begin{array}{rrr}
482.0 & -242.0 & 0 \\
-242.0 & 511.0 & -269.0 \\
0 & -269.0 & 269.0
\end{array}\right]
\end{aligned}
$$

Fig. 7 compares the simulated and experimental records for the absolute acceleration of floors 1 and 3 due to an initial displacement without the control logic in operation. In the experiment, the top floor was statically displaced approximately 3 in and released. The displacements from the static deflection of the

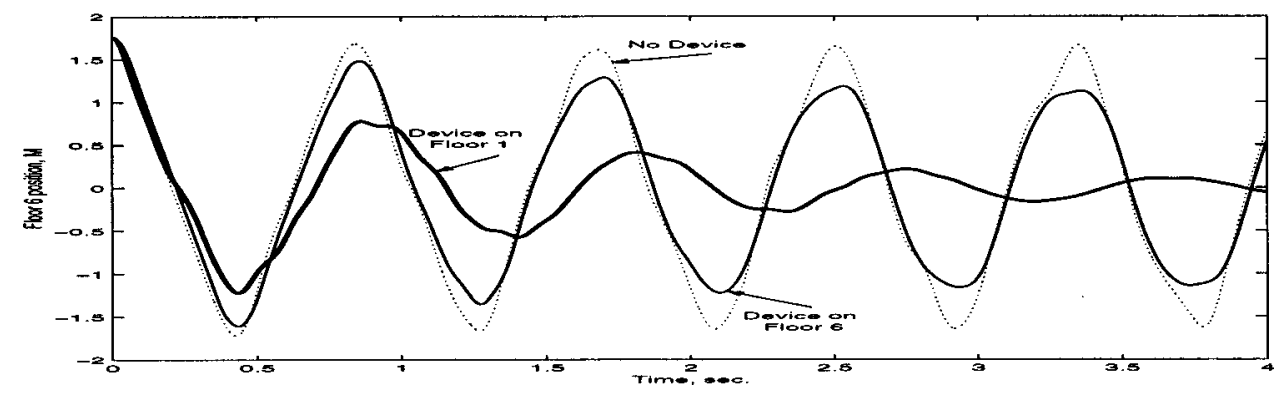

Fig. 12. Comparison of top floor displacement with resettable device located on bottom and on top floor 


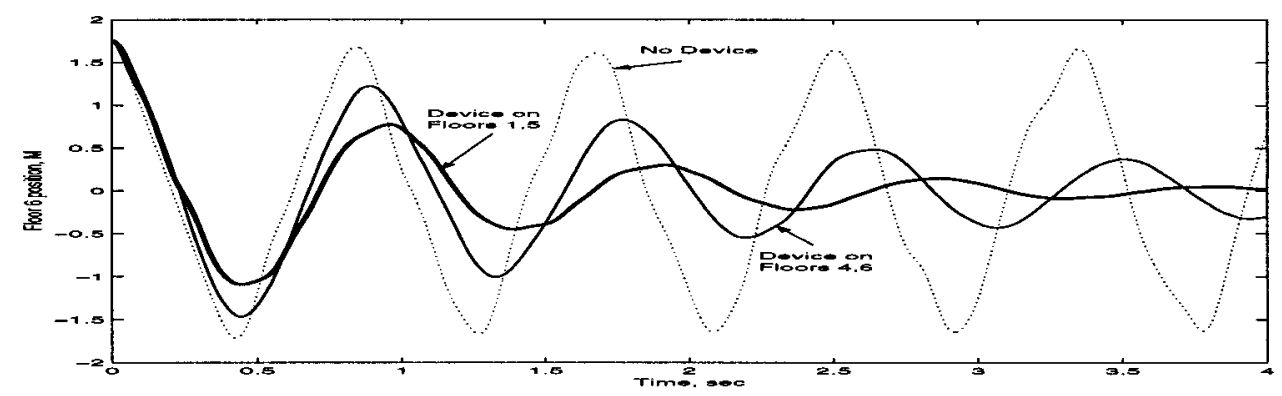

Fig. 13. Comparison of top floor displacement two devices located on floors one and six and on floors four and six

three floors due to the experimental initial condition were used as the initial conditions for the simulation. As the figure shows, the model seems to have a slightly different first natural frequency, leading to the behavior seen after ten or so cycles. Overall, however, the model has a reasonable degree of fidelity. Recall that the control logic does not depend on the structural model, but a reasonably accurate model can be used to study placement of the devices (more on this further below).

A pneumatic device (see left-hand side of Fig. 2) was installed on the first floor as shown in Fig. 6. The device adds roughly the same stiffness to the first floor as the existing structure has. This, as a result, increases the $(1,1)$ term of $K$ to $K_{11}+K 1_{11}=714$. Fig. 8 shows the effect of the control law on the transient response of the structure with the same initial conditions used for the response of Fig. 7. The top plots are simulation results and the lower plots are experimental results. The acceleration plots on the left-hand side show that the structural vibrations have settled in about $1 / 4$ the time of the response shown in Fig. 7.

The right-hand plots of Fig. 8 show the resisting force in the device during the motion. The jumps in force correspond to times when the device is reset. The dashed lines in the lower-right plot indicate the actual command to the experimental valve that causes the resetting action. The sharper features in the upper plots of Fig. 8 are due to the fact that in the simulation it is assumed that the devices can be reset instantaneously. In the experimental results shown in the lower plots, the dynamics associated with the movement of the value and the gas flow (as shown in Fig. 5) result in less sudden changes. Nonetheless, the simulation results of the top plots are quite similar to the experimental results of the lower plots and capture the essential features.

While adding the device increases the overall stiffness of the structure, the improvement in the response is due to the energy extraction in resetting. To see that, consider the top left plot of Fig. 8. The dotted curve corresponds to the response of the structure when the device is locked at all times, highlighting the benefits of the resetting logic (leaving the device open-unlockedresults in a similar plot).

These results are consistent with results of Bobrow et al. (1995), where some preliminary results were presented for response under external disturbance. For example, consider Figs. 9 and 10, from Bobrow et al. (1995), where the forced response of the structure to a periodic forcing function caused by the unbalanced rotating load on the top floor. The large amplitude portions of the plots occur when no control is applied, and the valve is closed. The smaller amplitude portions of the plots occur when the resetting control law is used. The peak acceleration is reduced by about one half. We should note that no attempt was made to optimize the size of the device in relation to the structure.

\section{Discussion}

The results shown above are relevant to applications that allow the addition of semiactive devices to existing structures, such as bridges and building retrofit projects. In such cases, the flexibility to choose a location is limited. Often, the suppression of the first vibrational mode is a major consideration. The development of the "Device Placement" section applied to this problem leads to placement of the device on the first floor, though a thorough comparison is difficult since adding a device to different floors results in different stiffness properties, modes shapes, and natural frequencies.

On the other hand, in applications such as flexible space structures or new buildings, it may be possible to install devices in a variety of locations. Furthermore, the design can be based on certain overall stiffness characteristics, in which a portion of the stiffness is from the semiactive devices and the rest from traditional structural elements-thus incorporating smart devices while avoiding excessive stiffness and expense. Since this approach maintains the same overall stiffness (and natural frequencies and mode shapes), it is quite useful for a comparative study of device placement (discussed in "Device Placement").

Consider the schematic of Fig. 11, which represents a six-story structure. We use a shear-beam model for a full-scale six-story building with identical story units. The mass of each floor, and the stiffness and damping coefficients of each story unit are $m_{i}$ $=345.6 \mathrm{t}, k_{i}=340,400 \mathrm{kN} / \mathrm{m}$, and $c_{i}=2,937 \mathrm{kNs} / \mathrm{m}$, respectively. These values result in a first vibrational mode of $1.2 \mathrm{~Hz}$. Table 1 shows the values of $v_{i}^{T} q_{j}$, as a measure of the influence of the device on the $i$ th floor on the $j$ th mode. For example, if suppression of the first mode is the main consideration, the first column of this table shows that a device placed between the ground floor and the first floor has the most influence on the first mode of vibration. The second column shows that the same device strongly affects the second mode as well, but a device placed between floors four and five would have the greatest effect on the second mode. On the other hand, placement between floors four and six should probably be avoided for suppressing the first mode shape.

To see the advantages of proposed choices for the position of the devices, consider Figs. 12 and 13. In all plots, the initial displacement was the static deflection curve of the structure due to a $100,000 \mathrm{~N}$ force applied to the top floor. Naturally, this excites primarily the lowest modes. The dotted lines in Figs. 12 and 13 show the response of the system without applying the control logic to any of the devices (i.e., nominal stiffness), and assuming zero damping. Fig. 12 shows that if a device is placed between the ground floor and the first floor (the thick line), a relatively fast 
settling time is achieved. On the other hand, as predicted by Table 1 , if a device is placed between floors five and six, a slower settling time is obtained. Fig. 13 compares the placement of two devices in two locations. If they are placed on the first and fifth floors (thick line), much faster settling times are achieved than by placing the devices on floors four and six. Also note that both plots, particularly Fig. 13, show that when a device is placed in the first floor, the first natural frequency changes slightly (leading to the mismatch in the phase after a few cycles).

\section{Conclusions}

This paper is focused on the basic analytical techniques needed to characterize structural systems that use a new type of "resettable" semiactive device for vibration suppression. The device behaves like a linear spring with an unstretched length that can be changed - or reset - at appropriate times to extract energy from the vibrating structure. We have presented experimental results on the mathematical characterization of the device, and we have presented both experimental and simulation results that demonstrate the performance of the device for structural vibration suppression. The results indicate that the device yields excellent performance, it is simple to construct, and it may easily be integrated into the design of new structures. It is also straightforward to retrofit the device into existing structures.

\section{Acknowledgments}

This research was supported in part by a grant from the National Science Foundation (CMS-9615731). The writers also wish to thank the associate editor and the anonymous referees for their help in the review process.

\section{References}

Bobrow, J. E., Jabbari, F., and Thai, K. (1995). "An active truss element and control law for vibration suppression." Smart Mater. Struct., 4, 264-269.

Bobrow, J. E., Jabbari, F., and Thai, K. (2000). "A new approach to shock isolation and vibration suppression using resettable actuators." ASME J. Dyn. Syst., Meas., Control, 122, 570-573.

Carlson, J. D., Catanzanite, M. D., and St. Clair, K. A. (1995). "Commercial magneto-rheological fluid devices." 5th Int. Conf. on ElectroRheological, Magneto-Rheological Suspensions and Associated Technology, Shefield, England.

Dyke, S. J., Spencer Jr., B. F., Sain, M. K., and Carlson, J. D. (1996). "Seismic response reduction using magneto-rheological dampers."
Proc., 1996 IFAC 13th Triennial World Congress, San Francisco, $145-150$.

Gavin, H. P. (1997). "The effect of particle concentration in homogeneities on the steady flow of electro- and magneto-rheological materials." J. Non-Newtonian Fluid Mech., 71(2), 165-182.

Gordaninejad, F., and Breese, D. G. (1999). "Heating of magnetorheological fluid dampers.” J. Intell. Mater. Syst. Struct., 10(8), 634645.

Inaudi, J. A., Leitmann, G., and Kelly, J. M. (1994). "Single degree of freedom nonlinear homogeneous systems." J. Eng. Mech., 120(7), 1543-1562.

Khalil, H. K. (1996). Nonlinear Systems, 2nd Ed., Prentice-Hall, Englewood Cliffs, N.J.

Kobori, T., and Kamagata, S. (1991). "Dynamic intelligent buildings: Active seismic response control." Intelligent structures; monitoring and control, Y. K. Wen, ed., Elsevier Applied Science, New York, 279-282.

Leitmann, G., and Reithmeirer, E. (1993). "Semiactive control of a vibrating system by means of electro-rheological fluids." Dyn. Control, 3(1), 7-34.

McClamroch, N. H., and Gavin, H. P. (1995). "Closed loop control using electro-rheological dampers." Proc., 1995 ACC, 4173-4177.

Nagarajaiah, S. (1997). "Semi-active control of structures." Proc., Structural Congress XV, 1997, ASCE, Portland, Ore., 1574-1578.

Nemin, D., Lin, Y., and Osegueda, R. (1994). "Semi-active motion control using variable stiffness." J. Struct. Eng., 120(4), 1291-1306.

Onodo, J., and Minesugi, K. (1996a). "Alternative control logic for type-II variable stiffness system." AIAA J., 34(1), 207-209.

Onodo, J., and Minesugi, K. (1996b). "Semiactive vibration suppression by variable damping members." AIAA J., 34(2), 355-361.

Patten, W. N., Mo, C., Kuehn, J., Lee, J., and Khaw, C. (1996). "Hydraulic semiactive vibration absorbers (SAVA): Separating myth from reality." Proc., 1996 International Federation of Automatic Control 13th Triennial World Congress, San Francisco, 157-162.

Patten, W. N., and Sack, R. L. (1994). "Semiactive control of civil engineering structures." Proc., 1994 ACC, Baltimore, 1078-1082.

Slotine, J. J. E., and Li, W. (1991). Applied nonlinear control, PrenticeHall, Englewood Cliffs, N.J.

Spencer, B. F., Dyke, S. J., Sain, M. K., and Carlson, J. D. (1997). "Phenomenological model for magnetorheological dampers." J. Eng. Mech. Div., Am. Soc. Civ. Eng., 123(3), 230-238.

Srisamang, R. E. (1997). "An experimental investigation of a new structural damping device." MS project, Univ. of California, Irvine, Calif.

Taylor, D. P., and Constantinou, M. C. (1995). "Testing procedures for high output fluid viscous dampers used in building and bridge structures to dissipate seismic energy." Shock Vib. Dig., 2(5), 373-381.

Thai, K. (1997). "Parametric methods for vibration suppression." $\mathrm{PhD}$ dissertation, Univ. of California, Irvine, Calif.

Utkin, V. I. (1997). "Variable structure systems with sliding modes." IEEE Trans. Autom. Control, 22(2), 212-222.

Yang, J. N., and Agrawak, A. K. (2001). "Semi-active hybrid control systems for nonlinear buildings against nearfield earthquakes." $J$. Eng. Struct., in press. 